AC 2010-1075: THE PERFORMANCE OF U.S. EXCHANGE STUDENTS ABROAD, A LONGITUDINAL STUDY

Joerg Mossbrucker, Milwaukee School of Engineering 


\title{
The Performance of U.S. Exchange Students Abroad, A Longitudinal Study
}

\begin{abstract}
The Milwaukee School of Engineering (MSOE) and the University of Applied Sciences Lübeck/ Germany (FHL) have a well-established, dual-accredited, bi-directional exchange program in Electrical Engineering that is completely integrated into the curricula of both institutions. The basic layout of this tuition-neutral exchange program is shown in Figure 1. MSOE and FHL students spend the first two years at their home institution. During the first half of the second year, the students express their desire to participate in the exchange program. After fulfilling all academic requirements, students from both universities spend their third (Junior) year together at FHL and their fourth (Senior) year together at MSOE. The full academic year abroad provides the best aspects of an immersion type program, but in the company of fellow students from one's home institution. All courses taken abroad are in the English language and fully transfer ensuring no delay of the students time to graduation. Upon successful participation students receive accredited undergraduate Electrical Engineering degrees from both institutions. Over 180 students have participated successfully in this award winning ${ }^{1}$ program since its inception 15 years ago with a success rate exceeding $98 \%$.
\end{abstract}

This paper is a longitudinal study of the academic performance of the U.S. students before, while, and after participating in this exchange program. Specifically, it tracks the student's performance in a number of technical and non-technical categories and demonstrates the effects of the ongoing course and program-level improvement process implemented between MSOE and FHL.

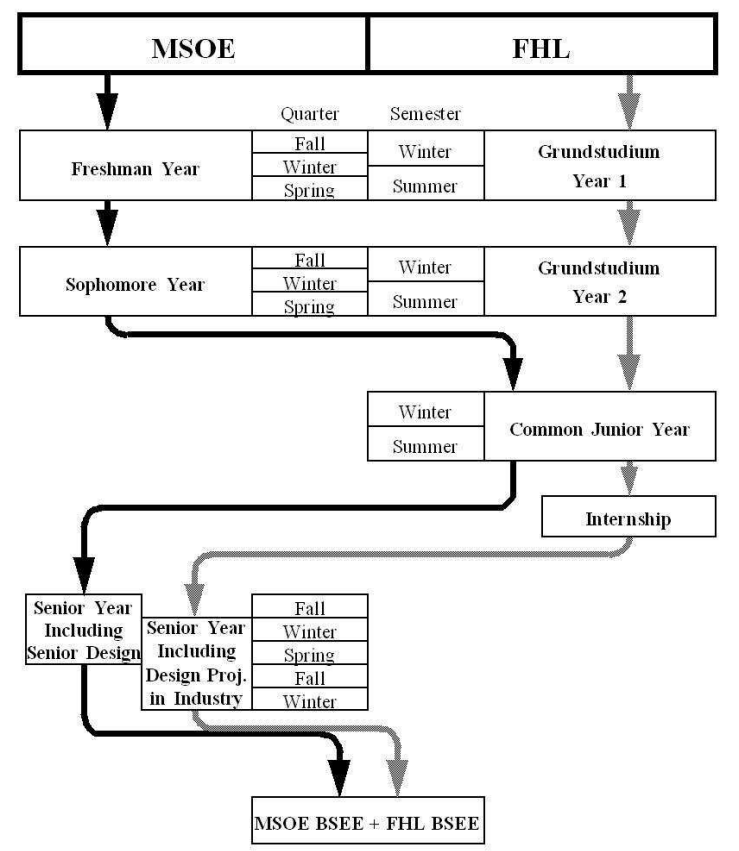

Figure 1 - Layout of the Exchange Program in Electrical Engineering 


\section{Participation}

The success of every exchange program depends first and foremost on student participation. If the total number of participating students per year is higher than a certain threshold, logistical measures have to be put into place to ensure smooth and (almost) seamless transfer of students. Once these logistical resources are present, the success of an exchange program relies on the constant stream of participating students. Students very often carefully weigh attracting factors, such as being abroad for almost an entire year, cultural immersion and horizon expansion, dual accredited degrees, added bonus for employment, with detracting factors, such as added financial costs and uncertainties about being abroad. In addition to that, participation numbers directly depend on total enrollment numbers, since exchange students in this particular program are a subset of the total student population of both institutions. This naturally leads to varying participation numbers, as evident in Figure 2, showing the number of MSOE and FHL students participating in the exchange program since its inception in 1994. This graph clearly shows widely varying student participation from both institutions. However, smoothing the data by taking a floating 3-point average as pictured in Figure 3 shows an altogether upward trend in the participation of MSOE students. In contrast, participation numbers of FHL students show a different trend. Between 1999 and 2005, participation of FHL students declined dramatically, mostly due to declining total enrollment numbers and economic factors ${ }^{2}$. However, since 2003, Freshmen enrollment at the FHL increased steadily ${ }^{3}$ and hence FHL participation in the exchange program again shows an upward trend.

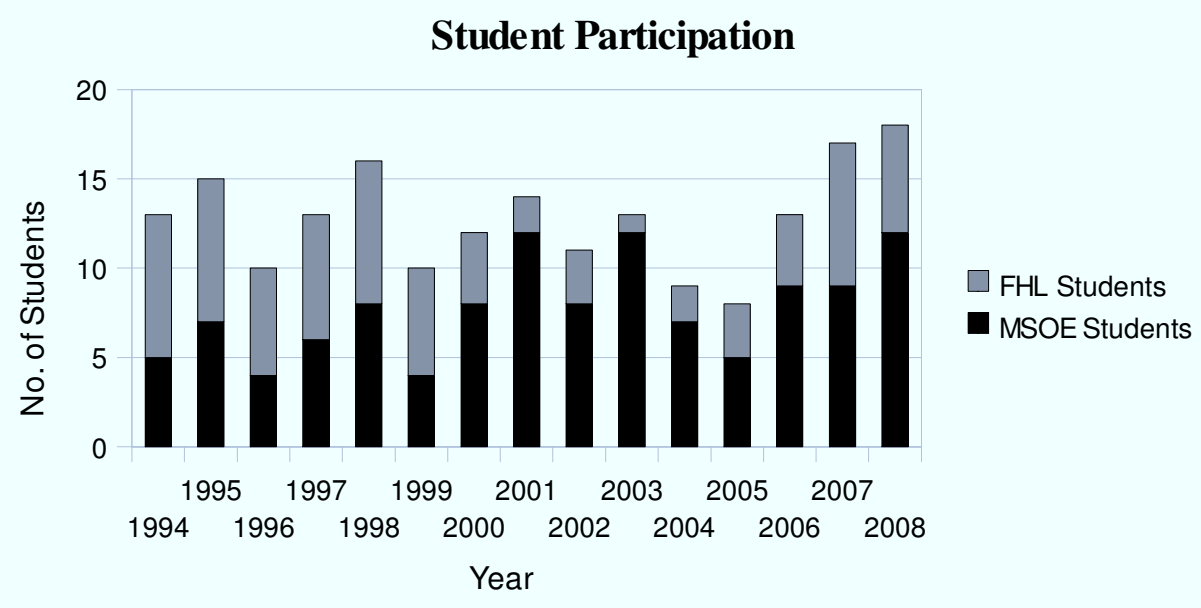

Figure 2 - Student Participation 


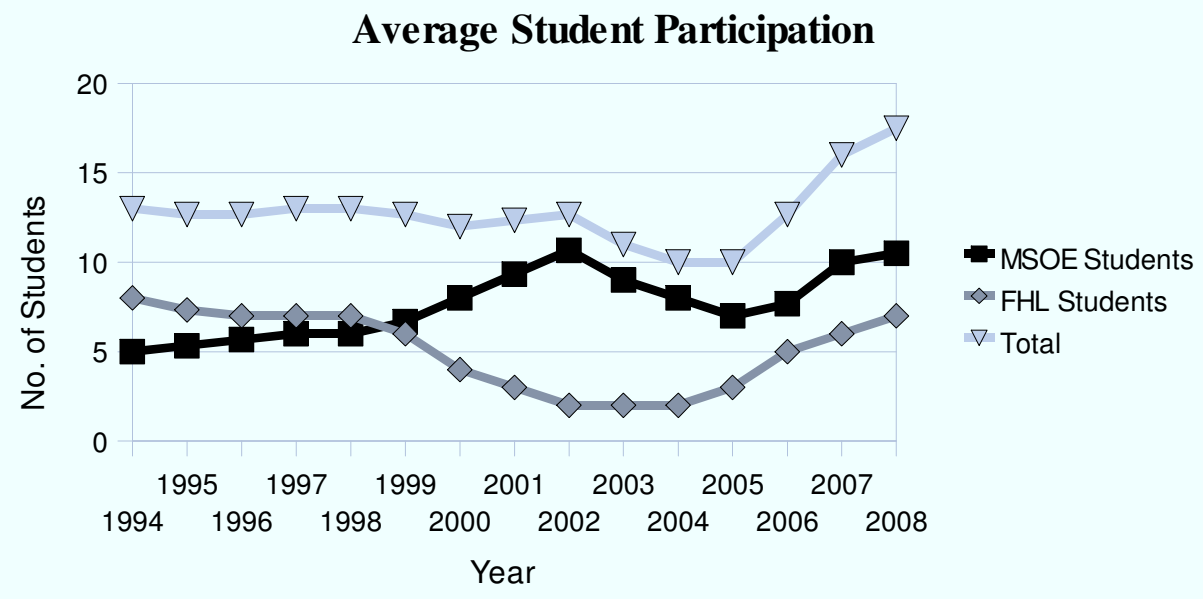

Figure 3 - Floating Average of Student Participation

\section{Grade Comparisons}

Grading scales and schemes differ considerably between MSOE and FHL. The grading scale at MSOE uses a typical U.S. grading scheme and is divided into 8 levels ${ }^{4}$ - A, AB, B, BC, C, CD, $\mathrm{D}$ and $\mathrm{F}$ (arranged best to worst). Contrastingly, the grading scale at FHL is using the mandated German grading scheme and is divided into 13 levels $^{5}-1.0,1.3,1.7,2.0,2.3,2.7,3.0,3.3,3.7$, 4.0, 4.3, 4.7, and 5.0 (arranged best to worst). Obviously, there is a need for grade conversion. Any grade conversion has to take into account definition and number of grade levels. Also, any type of conversion will most likely be non-linear, since grade definitions between the two education systems vary considerably ${ }^{4,5}$. The grading conversion chosen for the exchange program is shown in Figure 4. This conversion scheme was selected after extensive discussions between faculty of both institutions and careful comparison of grade definitions. The two highest FHL numerical grades of 1.0 and 1.3 translate into the highest MSOE numerical grade of 4.0 (an A), while the three lowest FHL numerical grades of 4.3, 4.7, and 5.0 - all considered to be nonpassing grades in the German education system ${ }^{5}$ - translate into the single MSOE failing grade of 0 (an F).

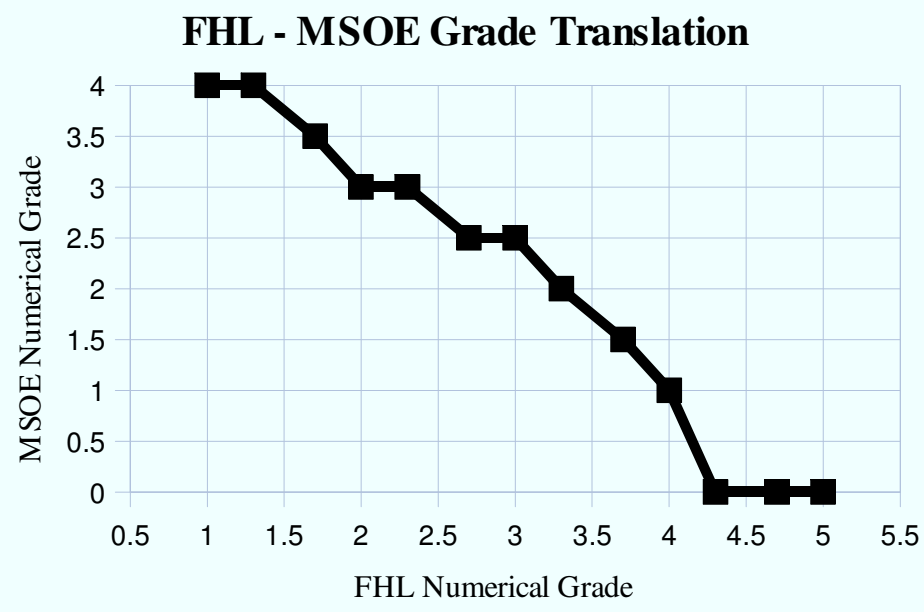

Figure 4 - FHL - MSOE Grade Translation 


\section{Overview of Data}

The academic performance of MSOE exchange students has been collected since the program's inception in 1994. The performance data consists of course-level grade-book entries, Term GPA at MSOE and FHL, and Cumulative GPA at MSOE. The data then is compared to the data of non-exchange students at MSOE and FHL, respectively. This allows a one-to-one comparison between exchange and non-exchange students without the need of additional academic performance assessment measures and procedures. Since the curriculum of the exchange program underwent a major change in 2001, only cumulative data from 2003 onward is demonstrated here. This corresponds to data of $58 \mathrm{MSOE}$ and 31 FHL exchange students.

The Term GPA and Cumulative GPA of all MSOE exchange students is shown in Figure 5. The horizontal axis gives either the corresponding academic quarter at MSOE (F - Fall, W - Winter, S - Spring) for the Freshmen, Sophomore, and Senior year, or the corresponding semester at FHL (FHL1 - Winter Semester, FHL2 - Summer Semester). The vertical axis displays the numerical Term GPA and Cumulative GPA. The (almost) imperceptible change in Term GPA between the end of the Sophomore year at MSOE and the end of the Summer semester at FHL suggests, that the overall grading translation shown in Figure 4 is a realistic one, despite its non-linear characteristic.

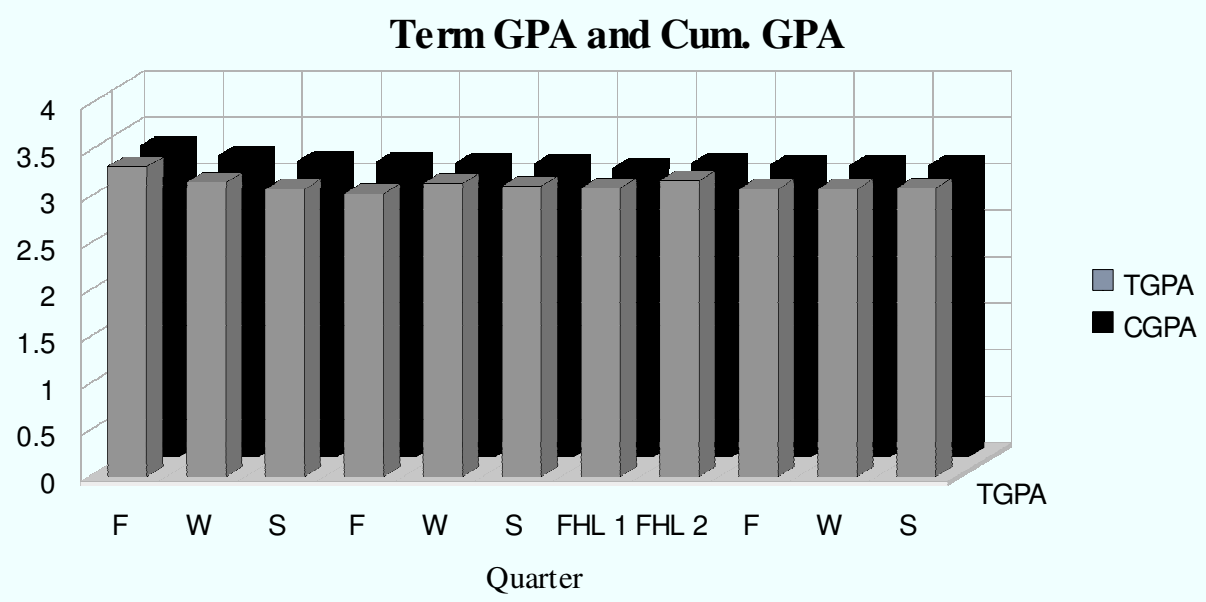

Figure 5 - Term GPA and Cumulative GPA of MSOE Exchange Students

The term GPA of the all MSOE exchange students have been divided into 5 ranges as shown in Figure 6. Again, the very small changes in student population within these five ranges between the end of the Sophomore year at MSOE and the end of the Junior year at FHL suggests, that the grade translation within these five individual grade ranges is realistic. 
TGPA Ranges

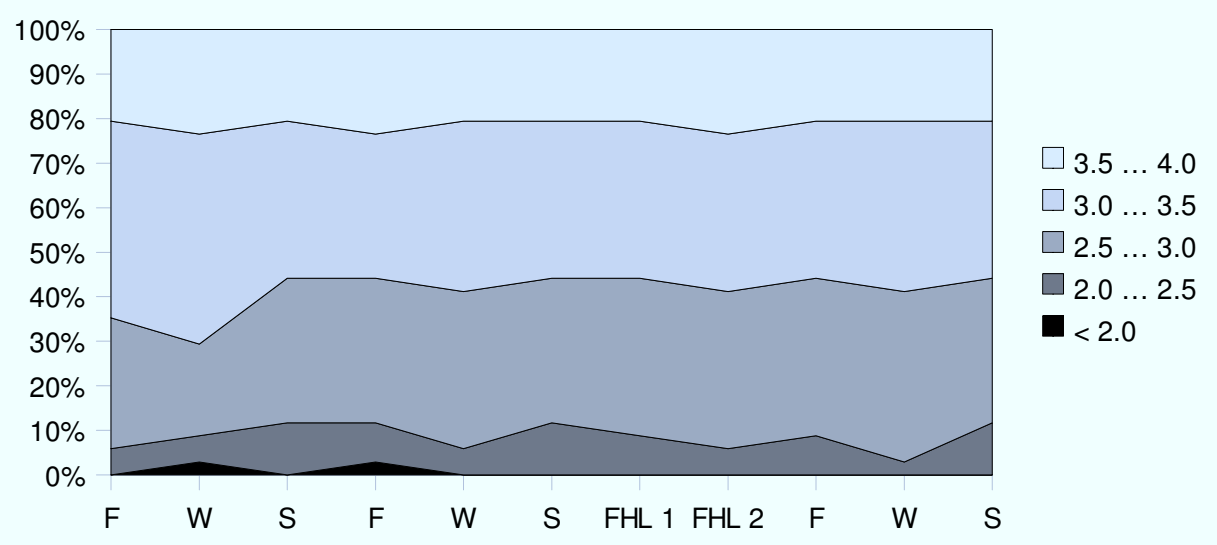

Figure 6 - Term GPA Ranges of MSOE Exchange Students

\section{Technical and Non-Technical Academic Performance}

The academic performance of MSOE exchange students has been divided into the performance in courses having technical content (such as Engineering and Natural Science courses) shown in Figure 7 and performance in courses having non-technical content (such as Humanities and Social Science courses) shown in Figure 8. Both figures show Term GPA during the years at MSOE and translated FHL Term GPA by comparing MSOE exchange and MSOE non-exchange students.

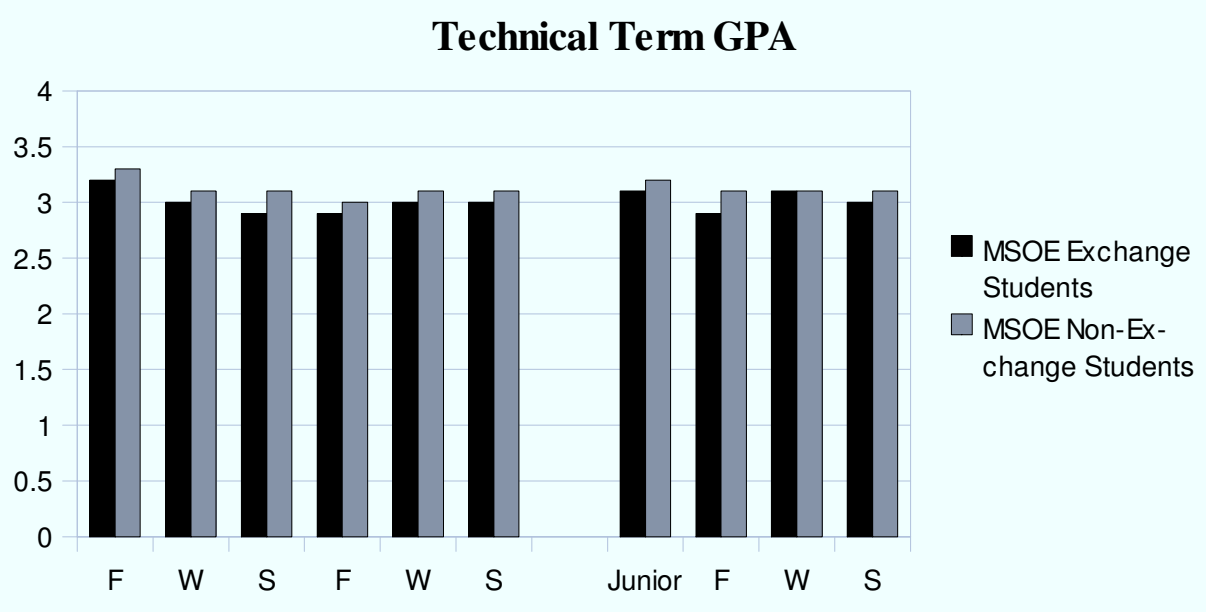

Figure 7 - Term GPA in Technical Courses 


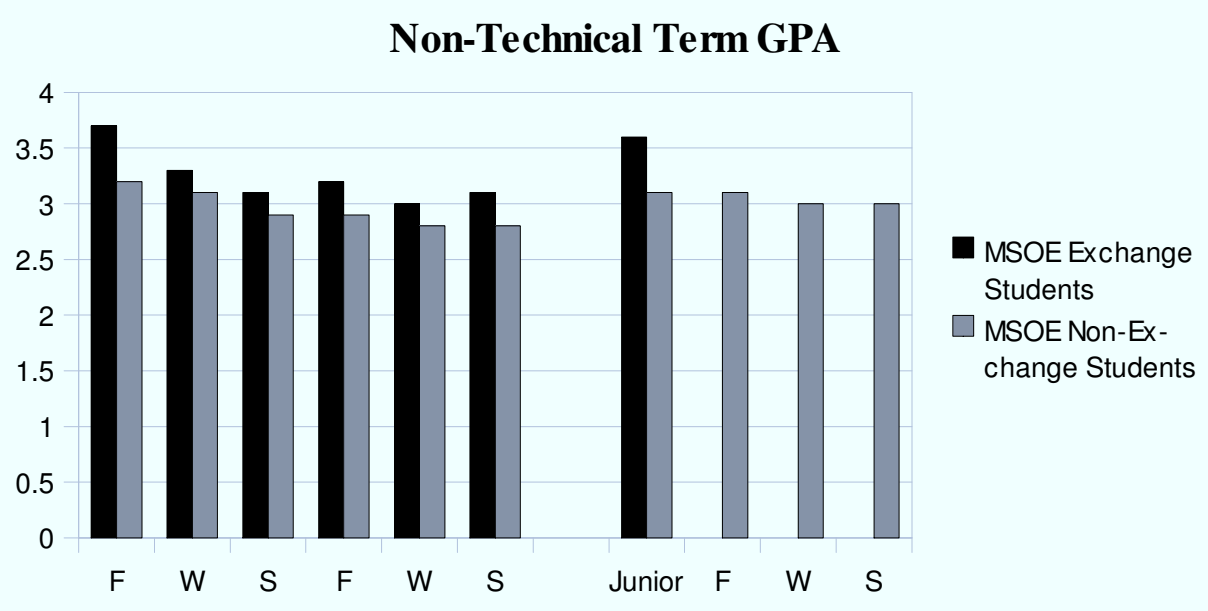

Figure 8 - Term GPA in Non-Technical Courses

Quite evident is the difference in the academic performance between exchange students and nonexchange students in both categories. The data suggests, that the "average" exchange student in engineering at MSOE might not be the "typical" engineering student, but rather one with an increased focus on the non-technical aspects (see also ${ }^{6}$ ). This is quite amplified at the end of the foreign experience (at the end of the Junior year), where the difference in performance in the non-technical area between exchange and non-exchange students is almost half of one grade (0.48).

\section{Performance at the FHL}

The academic performance of the MSOE exchange students at FHL has been compared with the academic performance of the FHL exchange students while at FHL. The data has been divided into three categories - overall term GPA, term GPA of courses not having a pre-requisite at MSOE, and courses having a pre-requisite at MSOE - and is shown in Figures 9, 10, and 11, respectively. The data suggests, that again the grade translation is realistic and that the preparation of MSOE exchange students for course taken abroad is at least adequate.

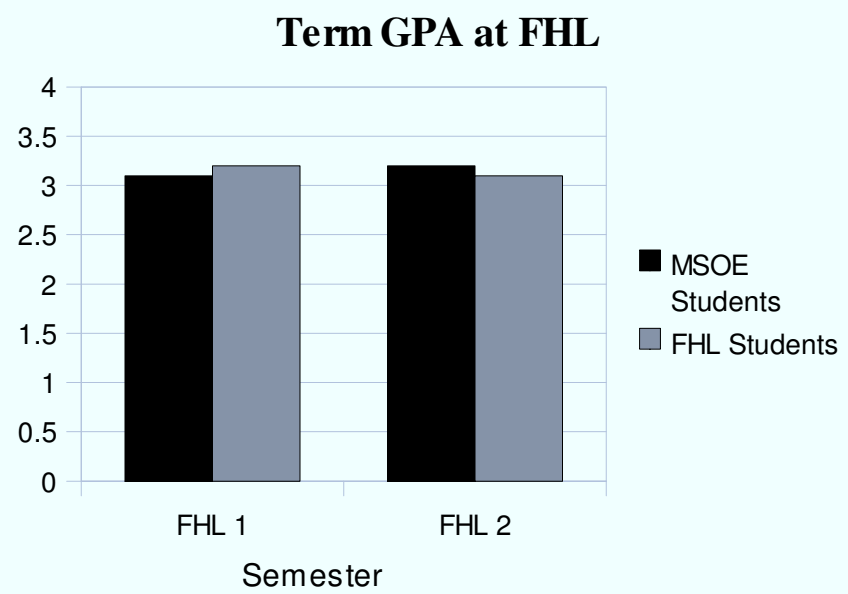

Figure 9 - Term GPA of MSOE Exchange Students at FHL 


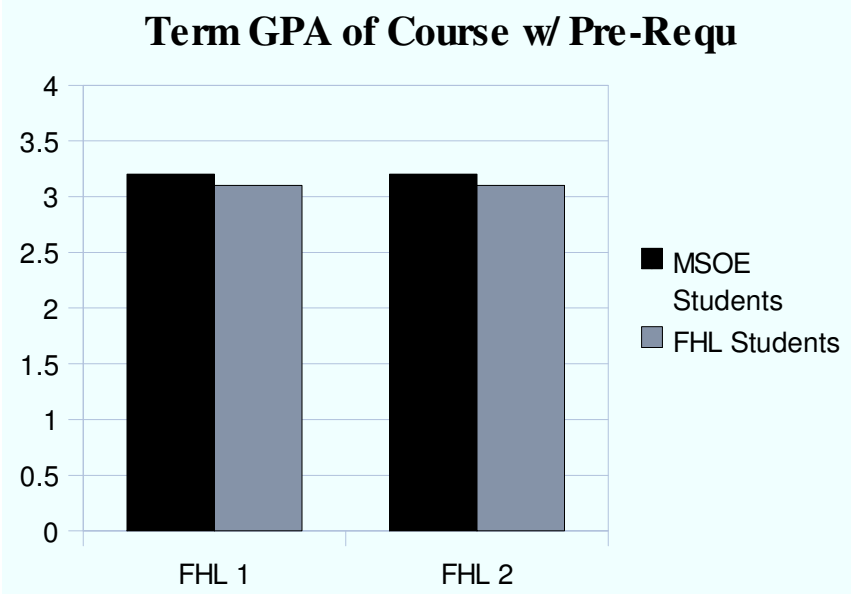

Figure 10 - Term GPA at FHL in Courses with Pre-Requ.

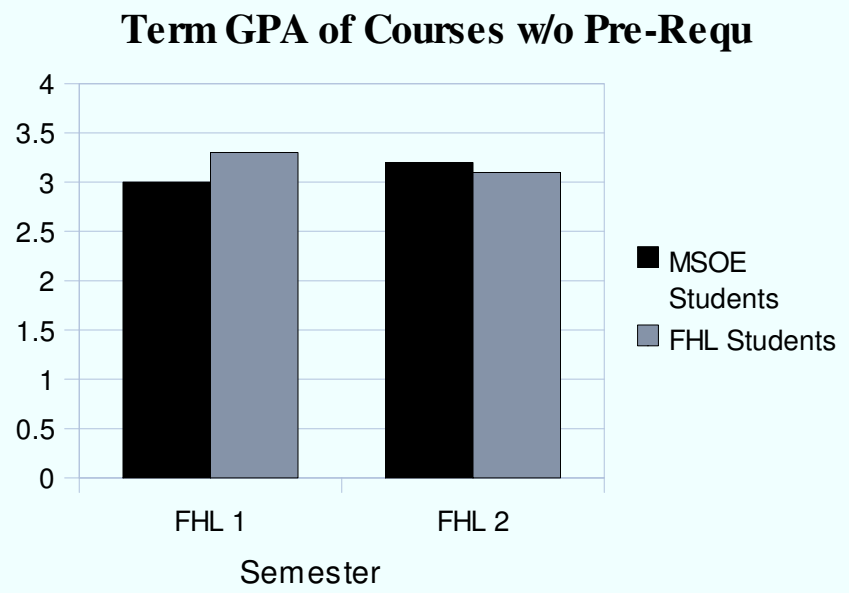

Figure 11 - Term GPA at FHL in Courses without Pre-Requ.

Also, quite evident is the so-called "culture-shock" - the difference in both education systems. The academic performance of MSOE exchange students significantly improves with the second semester abroad, as clearly seen in Figures 9 and 11.

Specifically, the academic performance in one course at FHL (Analog Electronics II) has been investigated, partly because of student feedback, but mostly because of a significant change in the pre-requisite course at MSOE. Figure 12 shows the average grade of MSOE exchange students in Analog Electronics II at the FHL. The horizontal axis shows the pre-requisite course at MSOE. This course changed from EE210 ${ }^{7}$, a general Analog Electronics course taken by students in Computer Engineering, to EE3111 ${ }^{7}$, a more specialized Analog Electronics I course taken by students in Electrical Engineering at MSOE. The data suggests, that this new prerequisite course prepares the students better than the previous one. 


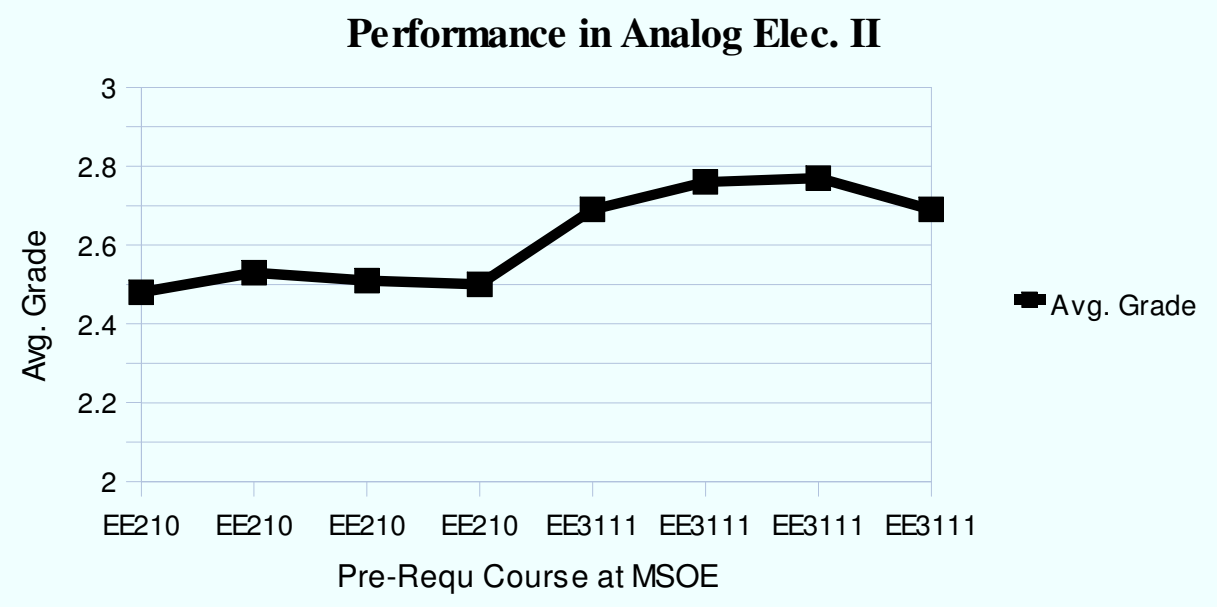

Figure 12 - Average Grade in Analog Electronics II at FHL

\section{Performance After Return to MSOE}

The academic performance of MSOE exchange students after their return to MSOE has been compared with the academic performance of MSOE non-exchange students. Due to the different curricula in the Senior Year, only the Senior Design courses EE407, EE408, and EE409 are taken by both student populations. The data is shown in Figure 13. It suggests, that exchange students experience what is called the "reverse culture shock". This also has been revealed in discussions between the author and the exchange students and faculty teaching Senior Design.

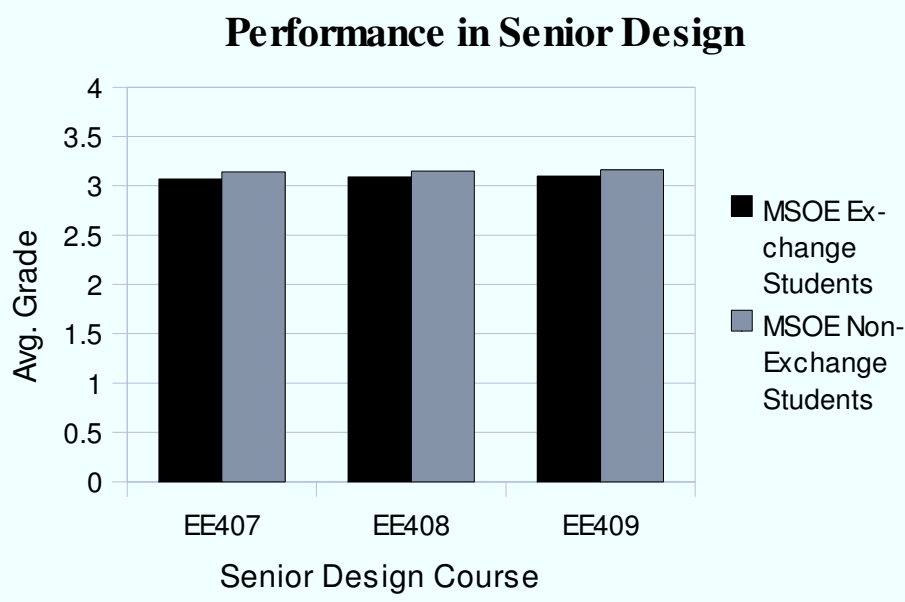

Figure 13 - Average Grade in Senior Design 


\section{Conclusions}

The Milwaukee School of Engineering and the Fachhochschule Lübeck/Germany have developed and implemented a student exchange program that is completely integrated into the curricula of both institutions. The academic performance of MSOE exchange students has been presented and the data analyzed. The data suggests that the implemented grading translation, necessary because of the quite different grading schemes used by both institutions, is accurate and fair. The data also suggests, that the exchange students at MSOE in Electrical Engineering have an increased focus on non-technical issues before going abroad and that the foreign experience is further amplifying this. Academic preparation for courses abroad is adequate and students seem to experience the typical so-called "culture shock" and "reverse culture shock".

\section{References}

[1] ECEDHA Innovative Program Award 2009: http://www.ecedha.org/awards/pastawardwinners.asp

[2] Report of the Rector: http://www.fhluebeck.de/Inhalt/09_Fachhochschule_intern/ 07_Services/04_Login01_Archive/03_Q_Medienarchiv/

04_Archive_der_Redakteure/Hoffmann_Roswitha/PDFs/Praesidium/bericht2003.pdf

[3] Report of the Rector: http://www.fhluebeck.de/Inhalt/09_Fachhochschule_intern/ 07_Services/04_Login01_Archive/03_Q_Medienarchiv/

04_Archive_der_Redakteure/Hoffmann_Roswitha/PDFs/Praesidium/bericht2005.pdf

[4] Course catalog at MSOE: http://www.msoe.edu/academics/course_catalog/undergrad2009-10.pdf

[5] Publication of University of Augsburg/Germany: http://www.uni-augsburg.de/en/programs/explanation/

[6] "VDE Ingenieurstudie 2005 - Elektro- und Informationstechnik", VDE publications, Germany

[7] Course descriptions can be found at the author's homepage at: http://myweb.msoe.edu/ mossbruc 